\title{
Verzeichnis der abgedruckten Entscheidungen
}

Der in dieser Entscheidungssammlung abgedruckte Text ist nicht immer mit dem Abdruck an den weiteren Fundstellen identisch. Die kursiv gedruckten Entscheidungen sind mit einer Anmerkung versehen.
I.1 RG, 27.4. 1905, 594/04 VI
I.2 RG, 13.2.1908, VI 172/07
JW 1905/368
1.3 RG, 27.5. 1910, II 409/09
JW $1908 / 236$
I.4 RG, 12.4. 1912, II 86/12
JW 1910/748
JW 1912/682;
LZ 1912 Sp. 1771
$1.5 \quad$ RG, 25. 2. 1915, VI 526/14
RGZ 87/1
I.6 RG, 19.4. 1916, VI $47 / 16$
LZ 1916 Sp. 1025
I.7 RG, 19.4. 1918, II 478/17
Recht 1918 Nr. 1363
I.8 RG, 29.4. 1918, VI 58/18
Recht 1918 Nr. 1367
I.9 RG, 6. 11. 1919, VI 215/19
RGZ 97/116
I.10 RG, 22. 12. 1919, VI 208/19
Recht 1920 Nr. 2845
I.11 RG, 29. 4. 1920, VI 6/20
Recht 1920 Nr. 2849
I.12 RG, 4. 1. 1921, II 374/20
RGZ 101/157
I.13 RG, 21.9. 1923, III $569 / 22$
RGZ 108/221
I.14 RG, 26. 6. 1929, I 17/29
RGZ 125/76
I.15 RG, 10.2. 1933, II 268/32
LZ 1933 Sp. 659
I.16 RG, 17. 1. 1940, II 82/39
RGZ 163/21
I.17 RG, 3. 4. 1940, II 148/39
DR $1940 / 1293$
I.18 BGH, 25.10. 1951, III ZR 95/50
BGHZ 4/1
I.19 BGH, 23. 6. 1952, III ZR 168/51
I.20 BGH, 1.4. 1953, VI ZR 77/53
I.21 BGH, 16. 12. 1953, VI ZR 12/53
I.22 BGH, 5. 11. 1955, VI ZR 199/54
I.23 BGH, 15. 3. 1956, II ZR 284/54
I.24 BGH, 21.4. 1956, VI ZR 36/55
I.25 BGH, 25.4. 1956, VI ZR 34/55
I.26 BGH, 13. 7. 1956, VI ZR 223/54
I.27 BGH, 29. 10. 1956, II ZR 79/55
VersR 1952/357 (Rungen- verschluß)
VersR 1953/242 (Speiseöl)
VersR 1954/100 (Trinkmilch)
VersR 1955/765, BB 1955/
1109 (Insektenvernichtungs- mittel)
VersR 1956/259 (Motorroller)
VersR 1956/410 (Fahrradgabel)
VersR 1956/419, NJW 1956/
1193 (Dreschmaschine)
VersR 1956/625 (Karussell)
BGHZ 22/90 
I.28 BGH, 17. 5. 1957, VI ZR 120/56

I.29 BGH, 4. 6. 1957, VI ZR 145/56

I.30 BGH, 25.3. 1958, VIII ZR 58/57

I.31 BGH, 11.7.1958, VI ZR 158/57

I.32 BGH, 14. 10. 1958, VI ZR 183/57

I.33 BGH, 14. 10. 1958, VIII ZR 143/57

I.34 BGH, 28. 10. 1958, VI ZR 176/57

I.35 BGH, 17.2. 1959, I StR 618/58

I.36 BGH, 14. 4. 1959, VI Zr 94/58

I.37 BGH, 15. 5. 1959, VI ZR 109/58

I.38 BGH, 10. 8. 1959, VIII ZR 113/58

I.39 BGH, 20. 10. 1959, VI ZR 152/58

I.40 BGH, 29. 10. 1959, VII ZR 176/58

I.41 BGH, 5. 7. 1960, VI ZR 130/59

I.42 BGH, 8. 7. 1960, VI ZR 159/59

I.43 BGH, 18. 10. 1960, VI ZR $8 / 60$

I.44 BGH, 21. 2. 1962, VIII ZR 4/61

I.45 BGH, 22. 2. 1962, VII ZR 205/60

I.46 BGH, 5. 4. 1962, VII ZR 183/60

I.47 BGH, 24. 1. 1963, VII ZR 100/61

I.48 BGH, 30. 4. 1963, VI ZR 7/62

I.49 BGH, 20. 12. 1965, VIII ZR 220/63

I.50 BGH, 28. 2. 1967, VI ZR 14/65

I.51 BGH, 8. 3. 1967, VIII ZR 4/65

I.52 BGH, 5. 4. 1967, VIII ZR 32/65
VersR 1957/584 (Gelenkwellenschutz)

VersR 1957/585 (Bagger)

BB 1956/426 (Leim)

VersR 1958/672 (Ziegel)

LM Nr. 7 zu $§ 459$ Abs. 1

BGB (Fußbodenbelag)

VersR 1959/104 (Seilschloß)

BB 1959/473 (Zwischen-

stecker)

VersR 1959/523 (Seilhexe)

JZ 1960/124 (Rostschutz-

mittel)

(Maschinenfett)

VersR 1960/342 (Fußboden-

klebemittel)

VersR 1960/344

(Auftaugerät)

VersR 1960/855

(Kondenstopf)

VersR 1960/856 (Silo)

VersR 1960/1095 (Kühl-

anlage)

(Auto-Scooter)

VersR 1962/480 (Heizkörper)

BGHZ 37/94

NJW 1963/1148

VersR 1963/860 (Auftau-

Transformator)

VersR 1966/241, Betr.

$1966 / 147$

VersR 1967/498 (Plastik-

massebehälter)

JZ 1967/321 (Farben-

Grundstoff)

BGHZ 47/312, Betr. 1967/

944, JZ 1968/228 (Betonbe-

reitungsanlage) 
I.53 BGH, 21.6. 1967, VIII ZR 26/65

I.54 BGH, 17. 10. 1967, VI ZR 70/66

1.55 BGH, 8. 5. 1968, VIII ZR 62/66

I.56 BGH, 29. 5. 1968, VIII ZR 77/66

I.57 BGH, 25. 9. 1968, VIII ZR 108/66

I.58 BGH, 26. 11. 1968, VI ZR 212/66

I.59 BGH, 22. 10. 1969, VIII ZR 196/67

I.60 BGH, 7. 7. 1970, VI ZR 223/68

I.61 BGH, 9. 7. 1970, VII 70/68

I.62 BGH, 28. 9. 1970, VIII ZR 166/68

I.63 BGH, 28. 4. 1971, VIII ZR 258/69

I.64 BGH, 16.6. 1971, VIII ZR 69/70

I.65 BGH, 6. 10. 1971, VIII ZR 95/70

I.66 BGH, 9.11. 1971, VI ZR 58/70

I.67 BGH, 24. 11. 1971, VIII ZR 81/70

I.68 BGH, 25. 11. 1971, VII ZR 82/70

I.69 BGH, 16. 2. 1972, VI ZR 111/70

I.70 BGH, 18.4. 1972, VI ZR 168/70

I.71 BGH, 17. 5. 1972, VIII ZR 98/17

I.72 BGH, 5. 7. 1972, VIII ZR 74/71

I.73 BGH, 11. 7. 1972, VI ZR 194/70

I.74 BGH, 4. 10. 1972, VIII ZR 117/71
BGHZ 48/118, NJW 1967/ 1903 (Trevira)

NJW 1968/247, VersR 1967/

1199 (Schubstrebe)

BGHZ 50/200, NJW 1968/

1622 (Kleber)

NJW 1968/2238, JZ 1968/

742 (Dieselöl)

BGHZ 51/91, NJW 1969/269, BB 1969/12, VersR 1969/155

(Hühnerpest)

N/W 1970/383

JZ 1971/63 (Druckfehler)

BGHZ 54/236

BB 1970/1414, Betr. 1970/

2213, VersR 1971/80, JZ

1971/29 (Bremsen)

BB 1971/673 (Tanklastzug)

WM 1971/1121, BB 1971/

1173

BB 1972/13, Betr. 1972/85, VersR 1972/149 (Förderanlage)

NJW 1972/252 (Futtermittel)

Betr. 1972/233, VersR 1972/

274

VersR 1972/559 (Förderkorb)

VersR 1972/693

Betr. 1972/1335 (Propangas)

BGHZ 59/158, BB 1972/1069

(Fensterlack)

BGHZ 59/172, NJW 1972/2217,

BB 1972/1161, VersR 1972/

1075 (Kurznarkosemittel)

BGHZ 59/303, NJW 1972/

2300, VersR 1973/33 (Wasser-

versorgung) 
I.75 BGH, 14. 3. 1973, VIII ZR 137/71

I.76 BGH, 19.6. 1973, VI ZR 178/71

I.77 BGH, 23. 10. 1973, VI ZR 162/72

I.78 BGH, 6. 12. 1973, VII ZR 17/72

I.79 BGH, 20. 12. 1973, VII ZR 184/72

I.80 BGH, 29. 1. 1974, VI ZR 53/71

I.81 BGH, 6. 2. 1974, VIII ZR 12/73

I.82 BGH, 14. 5. 1974, VI ZR 48/73

I.83 BGH, 16. 5. 1974, VII ZR 214/72

I.84 BGH, 24. 6. 1974, VII ZR 254/72

I.85 BGH, 19.2. 1975, VIII ZR 144/37

I.86 BGH, 3. 6. 1975, VI ZR 192/73
NJW 1973/843 (Nottesta.

mentsmappe)

NJW 1973/1602, VersR 1973/

862 (Feuerwerkskörper)

VersR 1974/243

NJW 1974/272, BB 1974/104

VersR $1975 / 750$

DAR $74 / 128$

BB 1974/998, NJW 1974/

1503

BGHZ 62/323, NJW 1974/

1322

BGHZ 64/46, BB 1975/806, NJW 1975/824 (Haartonikum) BB 1975/1031, Betr. 1975/1404 (Spannkupplungen)
II.1 OLG Stuttgart, 10. 12. 1907

II.2 OLG Bremen, 11. 10. 1951, 2 W 208/51

II.3 OLG Frankfurt, 7.1. 1954, U 173/52

II.4 OLG Celle, 4. 5. 1957, 9 U 65/55

II.5 OLG Köln, 9. 12. 1963, 10 U 44/62

II.6 OLG Karlsruhe, 4. 3. 1964, 1 U 154/63

II.6a OLG Zweibrücken, 7. 12. 1965, 1 U 63/65

II.7 OLG Frankfurt, 2.6. 1967, 5 U 136/66

II.8 OLG Celle, 28. 1. 1970, 13 U 175/69

II.9 OLG Saarbrücken, 17.3. 1970, 2 U 193/66

II.10 OLG Celle, 4. 12. 1970, 8 U 113/68

II.11 OLG Hamm, 18. 1. 1971, 3 U 193/70

II.12 OLG Hamburg, 22. 6. 1971, 7 U 113/70

II.13 OLG Köln, 19. 10. 1971, 15 U 67/71

II.14 OLG Hamm, 23. 10. 1972, 22 U 127/27

II.15 OLG Düsseldorf, 24. 10. 1972, 4 U 68/72
OLGE $18 / 69$

NJW 1951/145

VersR 1958/404

VersR 1964/541

BB 1964/740

VersR 1969/1121

BB $1970 / 1417$

BB 1971/845

DAR 1972/16

NJW 1972/162

MDR 1973/224, Betr. 1973/325 
II.16 Kammergericht, 3. 11. 1972, 17 U 1137/72 BB 1973/169

II.17 OLG Stuttgart, 31. 10. 1973, 2 U 64/73

II.18 OLG Hamm, 8. 11. 1973, 7 U 45/73 NJW 1974/1090

II.19 OLG München, 26. 4. 1974, 19 U 4324/73 Betr. 1974/1059

II.20 OLG Düsseldorf, 23. 7. 1974, 4 U 20/74 BB 1974/1366

III.1 LG Hanau, 28. 2. 1955, 2 O 117/53 VersR 1955/785

III. 2 LG Lindau, 26. 4. 1955, OH 83/64 VersR 1955/428

III.3 LG Freiburg, 17.2. 1959, 20 40/57

III.4 LG Duisburg, 14. 2. 1963, $10038 / 57$

III.5 LG Heidelberg, 10.4. 1963, 2 O 196/60 BB 1963/670

III.6 LG Kleve, 26. 2. 1964, $20157 / 62$

III.7 LG Limburg, 18.6. 1969, 2 O 11/69 NJW 1969/1574

III.8 LG Düsseldorf, 26. 5. 1971, 5 O 363/70 VersR 1972/671,

III.9 LG Hagen, 21.3. 1972, 5 O 259/71

III.10 LG Köln, 13. 4. 1972, 2 O 124/71 NJW 1972/1580

III.11 LG Braunschweig, 14. 12. 1972, 17 O 253/69

III.12 LG Heidelberg, 25. 7. 1973, 3 S 28/73 DAR 1974/124

III.13 LG Saarbrücken, 2. 7. 1974, 100 111/73

III.14 LG Hannover, 12. 6. 1975, $210275 / 73$ 